\title{
Behavioral Effects of Multiple Adrenal Medullary Grafts in Longtailed Macaques
}

\author{
Mark Dubach \\ Department of Psychiatry \& Behavioral Sciences, and Regional Primate Research Center \\ University of Washington SJ-50, Seattle, WA 98195, USA
}

A simple quantitative assay has been developed for comparing the behavioral effects of a variety of intracerebral treatments on lesioninduced deficits, in a non-human primate model of Parkinson's disease 15/. The home-cage turning behavior of 24 young adult male longtailed macaques (Macaca fascicularis), unilaterally lesioned by intranigral 6-hydroxydopamine (6-OHDA) injections, was monitored continuously, around the clock, before and after implants to the neostriatum, and results were evaluated separately for each animal by timeseries analysis. The assay indicated that grafts which succeeded in treating larger striatal regions produced relatively greater behavioral effects.

The sheer size of the primate neostriatum constrains transplantation therapy for parkinsonism. A stereotaxic approach can counter with numerous grafts to multiple striatal parenchymal sites. For adrenal medullary autografts, this strategy requires many small pieces cut from a single gland to retain viability pending transplantation. In monkey hosts, viability and behavioral effect depended heavily on technique. Preparation of long, narrow "ribbons" and delivery without deformation, in 7 subjects, provided thousands of viable, tyrosine hydroxylaseimmunoreactive cells in 3-10 transplant sites, in evidence as long as 8 weeks after transplantation $12,3 /$. The use of narrower or shorter ribbons in some of these subjects decreased the proportion surviving, but permitted a wider distribution throughout the neostriatum /4/. Of 4-13 ribbons per subject, some remained in medium $>2.5 \mathrm{~h}$ before implantation, without losing viability. Various other methods of preparation and delivery, used in 17 other subjects, left little viable tissue, in 1-3 transplant sites per animal.
Specially adapted rotometer cages permitted 24-hour measurement of spontaneous activity and turning preference. Each monkey in its home cage wore a nylon jacket fastened by flexible cable to a device atop the cage that sensed rotational position and passed the information to a microcomputer. A turn was defined as any set of consecutive events summing to a net movement of at least 90 degrees in one direction. Spontaneous ipsilateral bias was induced by 6-OHDA lesions in 6 of the 7 subjects receiving ribbon grafts and in 16 of the 17 other subjects. A paired t-test showed a highly significant $(p<0.001)$ decrease in contralateral turning after the lesion in the 22 affected subjects. Ribbon grafts shifted the bias back toward normal in 4 of the 6 affected monkeys receiving ribbon grafts, but not in the other subjects. A ttest showed that the difference between mean turning preference before and after the graft was significantly greater $(p<0.05)$ for the 6 monkeys receiving ribbon grafts than for the other 16 monkeys, which received poorly surviving grafts.

Group statistics, however, overlook interindividual differences such as graft placement, pre-lesion behavior, ribbon size, post-lesion interval, and latency before onset of treatment effects. Some of these differences are inherent to the experiment. Published behavioral results to date of non-human primate transplants have never involved more than a few animals receiving even nominally similar treatments. A statistical approach that allows evaluation of singlesubject data was applied, namely, a time-series analysis, the autoregressive integrated movingaverage method (ARIMA), adapted from Box and Jenkins /1/. This method evaluates pretreatment data to detect significant sequence- 
dependency, and provides a best-estimated predicted level for subsequent values, with a 95\% confidence interval, based on the null hypothesis that the treatment will have no effect. If the real data fall beyond this interval, then the treatment presumably had some effect.

Examination of individual records by ARIMA indicated the significance of lesion and graft effects in individual animals. For the 16 monkeys that received poorly surviving grafts by earlier methods, turning preference was not significantly affected by the grafts, according to this assessment. Among recipients of ribbon grafts, animals with greater total lengths of viable cell masses showed greater reversals. Grafts in the first 2 subjects receiving ribbon grafts had no significant effect, and in fact tended to decrease contralateral turning even further; total graft length in these subjects was relatively short, and striatal distribution therefore restricted, in spite of substantial numbers of surviving cells. Grafts in the other 4 subjects had significant effects, of a magnitude roughly proportional to the total graft lengths, which was greater in all cases than for the first 2 subjects. Implants therefore appear to take effect behaviorally if widely distributed, and further research should focus on methods for providing catecholamine to as wide a striatal region as possible.

\section{REFERENCES}

1. Box GEP, Jenkins GM. Time Series Analysis: Forecasting and Control. San Francisco, CA: Holden Day, 1970.

2. Dubach M, German DC. Extensive survival of chromaffin cells in adrenal medulla "ribbon" grafts in the monkey neostriatum. Exp Neurol 1990; 110: 167-180.

3. Dubach M. Adrenal medulla "ribbon" grafts in nonhuman primates: Transplant methods. J Neurosci Meth 1991; 39: 19-28.

4. Dubach $M$. Viable adrenal medullary transplants in non-human primates: Increasing the number of grafts. J Neur Transplant Plast 1992; 3: 81-96.

5. Dubach $M$. Behavioral effects of adrenal medullary grafts in non-human primates. J Neur Transplant Plast 1992; 3: 97-114. 

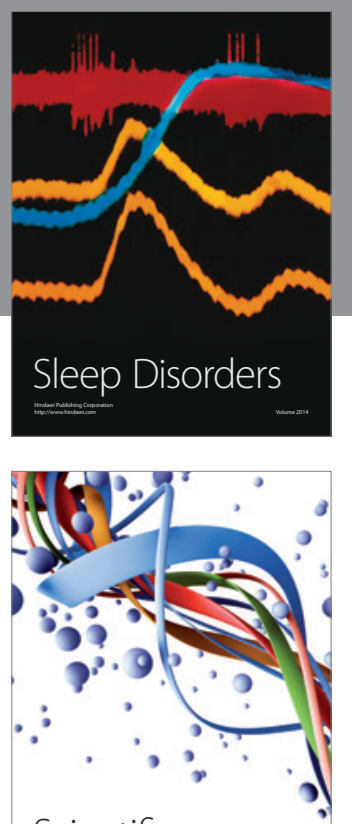

Scientifica
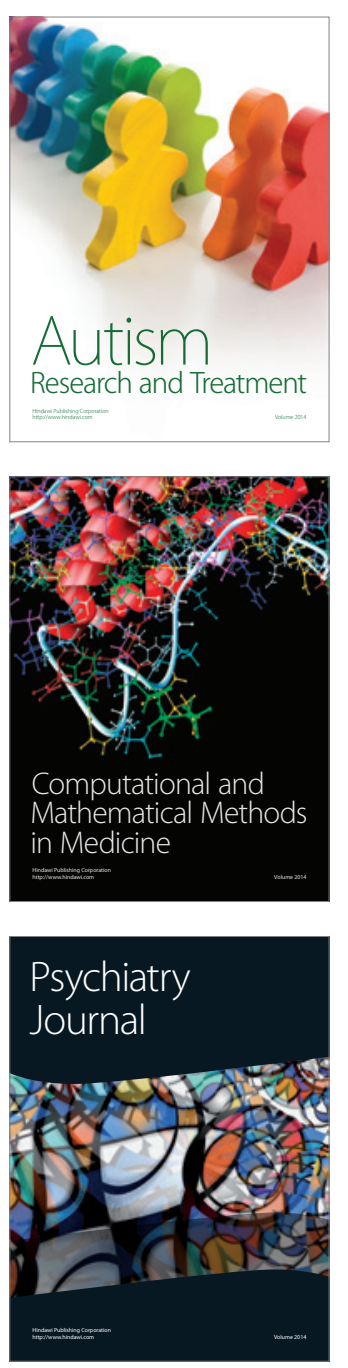
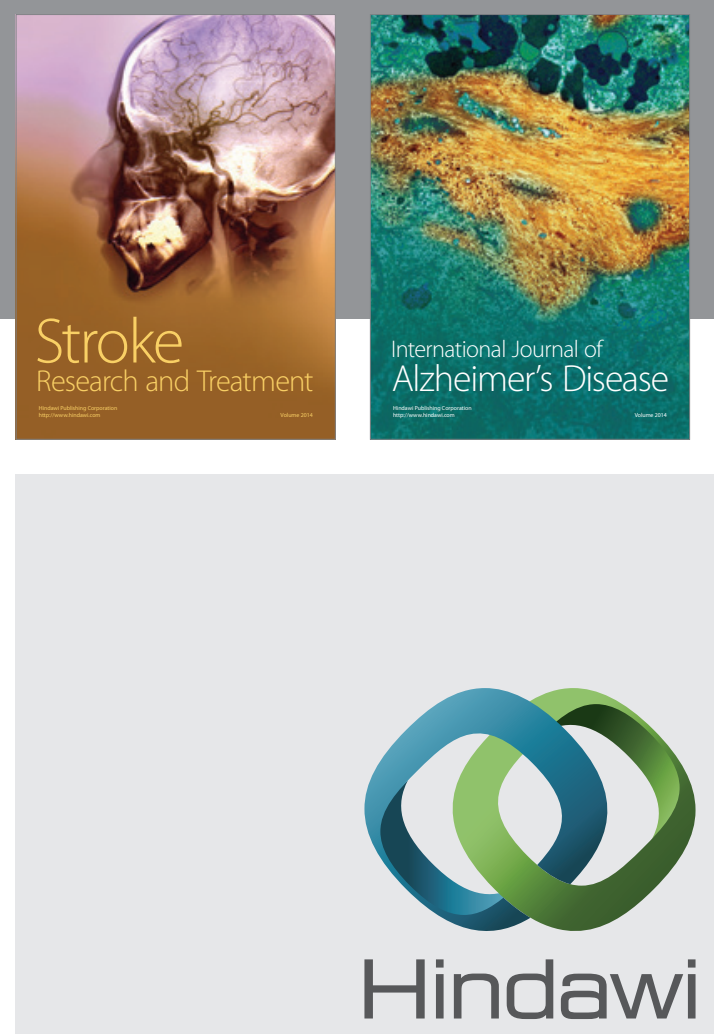

Submit your manuscripts at

http://www.hindawi.com
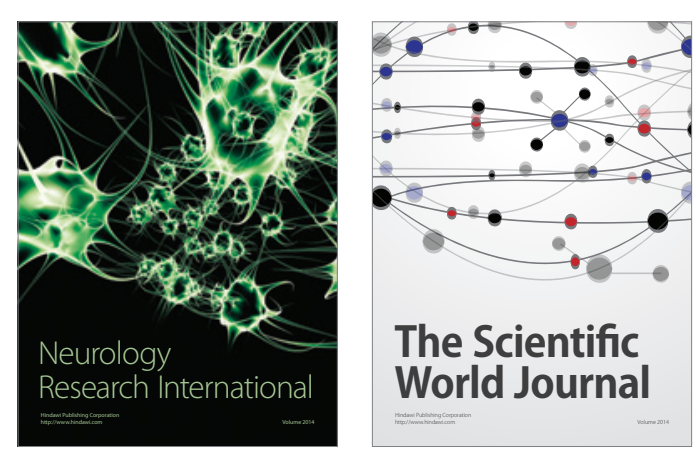

The Scientific World Journal

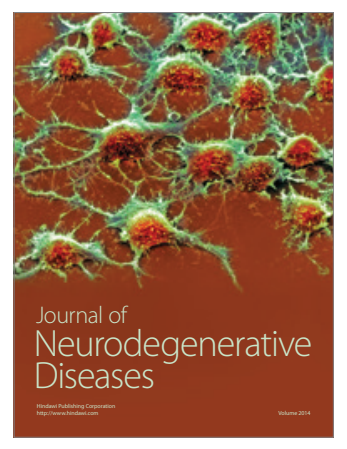

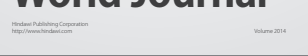

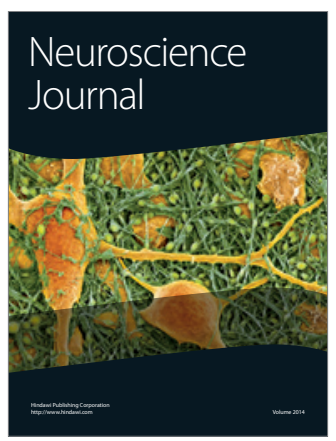

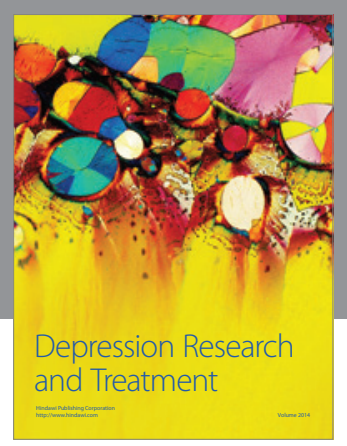
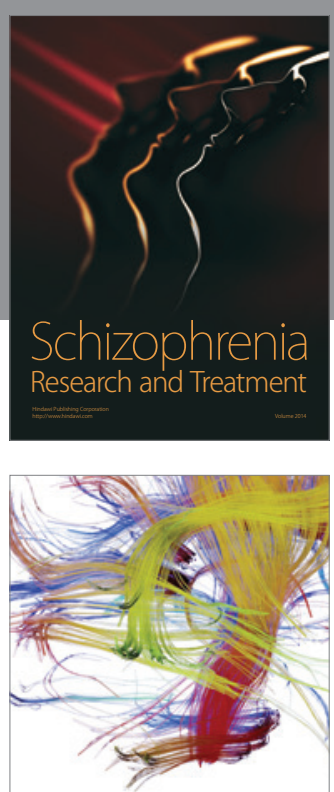

Brain Science

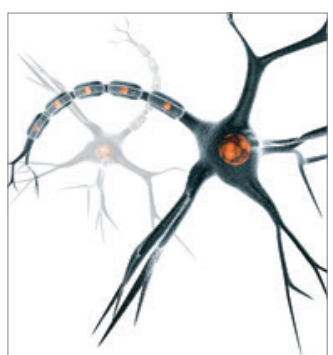

Neural Plasticity
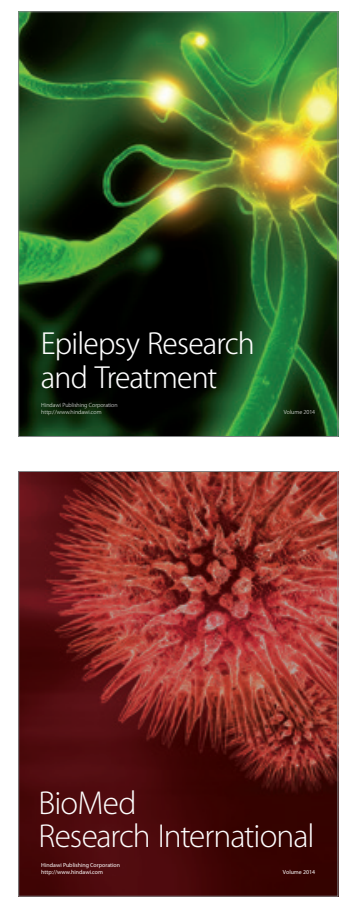

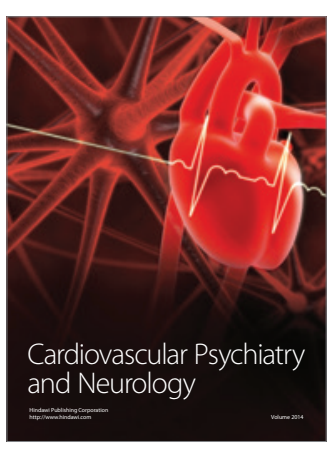

Parkinson's

Disease
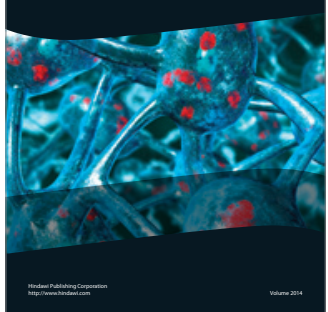